\title{
SUPRAPOSINORMALITY AND HYPONORMALITY FOR THE GENERALIZED CESÀRO MATRICES OF ORDER TWO
}

\author{
H. C. RHALY JR.
}

Abstract. It is well known that the generalized Cesàro matrices of order one are hyponormal operators on $\ell^{2}$, and it is also known that the Cesàro matrix of order two is hyponormal. Here the relatively new concept of supraposinormality is used to show that the generalized Cesàro matrices of order two are both posinormal and coposinormal, and that "most" of them are also hyponormal. A conjecture is propounded that would extend the hyponormality result.

Mathematics subject classification (2010): 47B20.

Keywords and phrases: Cesàro matrix, hyponormal operator, supraposinormal operator.

\section{REFERENCES}

[1] K. ENDL, Untersuchungen über momentenprobleme bei verfahren vom Hausdorffschen typus, Math. Ann. 139 (1960), 403-432.

[2] F. HAusdorfF, Summationsmethoden und momentfolgen. I., Math Z. 9 (1921), no. 1-2, 74-109.

[3] F. HAUSDORFF, Summationsmethoden und momentfolgen. II., Math Z. 9 (1921), no. 3-4, 280-299.

[4] A. JAKIMOVSKI, The product of summability methods: New classes of transformations and their properties, Tech. Note (4), Contract no. AF 61 (052)-187 (1959).

[5] C. S. Kubrusly, P. C. M. Vieira, And J. ZAnni, Powers of posinormal operators, Oper. Matrices 10 (2016), no. 1, 15-27.

[6] H. C. Rhaly JR., Posinormal operators, J. Math. Soc. Japan 46 (1994), no. 4, 587-605.

[7] H. C. RHALY JR., Remarks concerning some generalized Cesàro operators on $\ell^{2}$, J. Chungcheong Math. Soc. 23 (2010), no. 3, 425-433.

[8] H. C. RHALY JR., A superclass of the posinormal operators, New York J. Math. 20 (2014), 497-506. This paper is available via http://nyjm. albany.edu/j/2014/20-28.html.

[9] H. C. RHALY JR., The Nörlund operator on $\ell^{2}$ generated by the sequence of positive integers is hyponormal, Bull. Belg. Math. Soc. Simon Stevin 22 (2015), no. 5, 737-742.

[10] B. E. RHOADES, Generalized Hausdorff matrices bounded on $\ell^{p}$ and $c$, Acta Sci. Math. (Szeged) 43 (1981), 333-345.

[11] N. K Sharma, Hausdorff Operators, Acta Sci Math. (Szeged) 35 (1973), 165-167.

[12] W. A. Stein et AL., Sage Mathematics Software (Version 6.10), The Sage Developers, 2015, http://www. sagemath.org. 